\title{
Evaluation of phytochemical antioxidant levels by hydrogen peroxide scavenging assay
}

\author{
Ajla Pleh ${ }^{1}$, Lejla Mahmutović ${ }^{2}$ and Altijana Hromić-Jahjefendić ${ }^{3^{*}}$ \\ ${ }_{1,2,3}$ Genetics and Bioengineering, International University of Sarajevo, Bosnia and Herzegovina
}

*Corresponding author: ahromic@ius.edu.ba

\section{(C) The Author}

2021.

Published by

ARDA.

\begin{abstract}
The aim of this study was to determine the antioxidant capacity of some phytochemicals by using an $\mathrm{H}_{2} \mathrm{O}_{2}$ scavenging assay. Betaine, Allantoin and Nicotinamide were put to the test. Even though hydrogen peroxide $\left(\mathrm{H}_{2} \mathrm{O}_{2}\right)$ is not intrinsically reactive, it can be transformed into the extremely reactive and harmful hydroxyl radical (HO), which is then able to interact with nucleotides in deoxyribose nucleic acid and in that way trigger breakage of the strand resulting in carcinogenesis, mutagenesis, etc. Antioxidants aid in the protection of cells from the harmful effects of reactive oxygen species that are known to induce oxidative stress. Excessive production of these reactive oxygen species in the human body is associated with many chronic degenerative diseases such as diabetes, neurodegenerative disease, cancer, etc. The effective way to minimize levels of oxidative stress is the ability to scavenge these reactive oxygen species (ROS). Also, phytochemicals are able to act as antioxidants, and in that way play a vital role in the prevention of disease caused by oxidative stress. The ability of a compound to scavenge $\mathrm{H}_{2} \mathrm{O}_{2}$ is a good predictor of its potential antioxidant function. The hydrogen peroxide $\left(\mathrm{H}_{2} \mathrm{O}_{2}\right)$ scavenging assay was determined using the Ruch et al., 1989 method, and a UV-VIS spectrophotometer. In conclusion, our samples had marvellous $\mathrm{H}_{2} \mathrm{O}_{2}$ scavenging activity and possessed good antioxidant capability, and were compared with the ascorbic acid (vitamin c) as standard natural antioxidant/ as reference antioxidant. The samples were also able to scavenge $\mathrm{H}_{2} \mathrm{O}_{2}$ in a concentration-dependent way, according to our results.
\end{abstract}

Keywords: $\mathrm{H}_{2} \mathrm{O}_{2}$ scavenging assay, hydrogen peroxide, ROS, phytochemicals, antioxidants, betaine, allantoin, nicotinamide.

\section{Introduction}

\subsection{Reactive oxygen species}

ROS, or reactive oxygen species, refers to a wide range of compounds and free radicals emanated from molecular oxygen [1]. Free radicals including superoxide anion radicals $\left(\mathrm{O}_{2}{ }^{-}\right)$, hydroxyl radicals $(\mathrm{OH} \cdot)$, and non-free radical species including hydrogen peroxide $\left(\mathrm{H}_{2} \mathrm{O}_{2}\right)$ and singlet oxygen $\left({ }^{1} \mathrm{O}_{2}\right)$ are examples of ROS [2]. Free radicals can be formed from endogenous and exogenous sources. Endogenous can be inflammation, cancer, mental stress, infection, ageing. While exogenously free radicals can penetrate the body and degrade due to exposure to heavy metals, such as mercury, lead, iron, some environmental pollutants, or drugs

This work is licensed under a Creative Commons Attribution License (https://creativecommons.org/licenses/by/4.0/) that allows others (cc) (†) to share and adapt the material for any purpose (even commercially), in any medium with an acknowledgement of the work's authorship and initial publication in this journal. 
BES Vol. 2, No. 1, June 2021, pp.1-10

(cyclosporine, gentamicin), cigarette smoke, alcohol, radiation, cooking (smoked meat, used oil, and fat), consumption of food treated with pesticides, etc. In these ways, ROS gets generated inside our bodies [3]. Reactive oxygen species (ROS) are most typically formed by mitochondria. During mitochondrial electron transport, reactive oxygen species are produced as by-products [4]. Normal metabolism produces reduced $\mathrm{NADH}$, which contributes an electron to a complex one, which then gives electrons to complex three, which then donates an electron to complex four. Complex four obtains electrons and transfers them to oxygen, culminating in the production of water. However, since there are so many oxygen molecules inside the mitochondria, these oxygen molecules will occasionally collide with a complex one or complex three, causing this oxygen to prematurely receive electrons from the electron transport chain and then generate a superoxide radical, which is a free radical with an unpaired electron. In our cells, we have the superoxide dismutase enzyme (SOD), which takes the superoxide free radical and converts it to hydrogen peroxide [5]. Hydrogen peroxide is converted into safe water and oxygen with the help of catalase, and it can also be converted into hydroxyl radicals that are very reactive in the presence of transition metals such as iron. In Fenton's reaction (1) a hydrogen peroxide molecule receives one electron from soluble Fe(II) iron, allowing it to decompose and develops hydroxyl radical [6].

$$
\mathrm{Fe}(\mathrm{II})+\mathrm{H}_{2} \mathrm{O}_{2} \rightarrow \mathrm{Fe}(\mathrm{III})+\mathrm{OH} \cdot+\mathrm{OH}^{-}
$$

ROS may destroy important biomolecules including lipids, carbohydrates, and proteins, as well as DNA, causing mutations. They need to be scavenged successfully by constituents of cells, if not done so, they will cause many disease conditions. The immensity of reactive oxygen species stationed in the human body is commonly involved in a variety of processes that inflict cellular damage including cancer, neurodegeneration, ageing, atherosclerosis, ischemic injury, inflammation, Alzheimer's, Parkinson's diseases and diabetes [7]. To remove damaged molecules, and prevent the progression of diseases, the body natural's antioxidant defences, such as antioxidant enzymes should be enhanced or supplementation with food ingredients that contain antioxidants must be improved. Antioxidant defence system protects cells from ROS-induced damage relying on antioxidant enzymes such as superoxide dismutase (SOD), catalase (CAT), and glutathione peroxidase (GPx) [8]. They also offer protection against oxidative stress through scavenging free radicals, inhibiting lipid peroxidation, and preventing disease development through a variety of pathways. The discrepancy between the formation of reactive oxygen species and antioxidant defence is known as oxidative stress, which since it is associated with inflammation, is important in the pathogenesis of cancer and cardiovascular disease, obesity, and type 2 diabetes [9]. Antioxidant intake is essential to reduce oxidative stress and promote comprehensive human health according to many epidemiological together with in vivo studies [10]. $\mathrm{H}_{2} \mathrm{O}_{2}$ is commonly accepted as a cytotoxic agent and it must be reduced by antioxidant defence enzymes [11]. Hydrogen peroxide is all around us. We can find it in drinking water, in rainwater, seawater, even in human blood plasma. Various beverages, such as instant coffee, green and black tea in concentrations higher than $100 \mu \mathrm{M}$ contain hydrogen peroxide [12].

This study aimed to evaluate the ability of phytochemicals (betaine, allantoin and nicotinamide) to scavenge $\mathrm{H}_{2} \mathrm{O}_{2}$ (hydrogen peroxide) concentration. Further, we tried to analyze which of the phytochemicals tested demonstrates the highest antioxidant capability.

\subsection{Phytochemicals}

Phytochemicals, phytobiotics, and phytogenics are all terms for natural bioactive substances obtained from plants (,,phyto "coming from a Greek word meaning ,plant “). They are known as groups of compounds that belong to secondary plant metabolites and include broad-spectrum of chemical units such as polyphenols, flavonoids, alkaloids, organ sulfur compounds, a nitrogen-containing compound, steroidal saponins, and vitamins [13]. Phytochemicals act as antioxidants.

The inclusion of phytochemicals in the diet leads to the reduction of oxidative stress, neutralization of free radicals and increase of antioxidant activity in various tissues, resulting in an overall improvement of health. Most of the phytochemicals are encountered in a variety of vegetables, fruits, grains alongside other plants 
BES Vol. 2, No. 1, June 2021, pp.1-10

and possess a variety of health benefits, including the prevention and treatment of cancer pathologies, cardiovascular diseases, nerve disorders and Alzheimer's disease [14]

\subsubsection{Betaine}

Betaine (BET) is a natural chemical that has been extensively researched as an antioxidant in human health [15]. Betaine is a natural amino acid substance that is both stable and non-toxic, as well as very soluble in water. It is also called trimethyl glycine because it resembles glycine in appearance with three additional methyl groups. The zwitterionic quaternary ammonium form $\left[\left(\mathrm{CH}_{3}\right) 3 \mathrm{~N}+\mathrm{CH}_{2} \mathrm{COO}\right]$ is also present in betaine. Betaine was firstly detected in the plant known as Beta vulgaris (juice) in the 19th century [16].

Foods that contain betaine are spinach, shrimp, beets, whole grains, quinoa, brown rice, sweet potatoes, and shellfish, etc. [17]. According to research, betaine has been found to have positive effects on human diseases such as diabetes, cancer, obesity and Alzheimer's disease. Scientists have linked betaine intake to breast, lung, liver, colon and nasopharyngeal cancers. Studies have shown that the higher the betaine intake, the lower the risk of cancer [18]. Betaine is used both as an osmolyte and as a source of methyl gatherings, which helps to maintain the health of the liver, kidneys, and heart [19].

\subsubsection{Allantoin}

Allantoin is a metabolic intermediate used in a diverse variety of organisms, including bacteria, plants, and animals. It is found in plants, especially in the leaves and roots of the comfrey Symphytum officinale (from the Boraginaceae family). Allantoin is a purine-derived heterocyclic compound that derives from the oxidation of uric acid. It's an odorless white powder that's soluble in water but not so much in alcohol and completely insoluble in oils [20]. In plants such as comfrey, chamomile, sugar beet, tobacco seeds and wheat germ, aloe vera we find allantoin. Allantoin is generated in cells from uric acid. According to the research, it has the capacity to scavenge free radicals, reduce inflammatory markers, and induce collagen [21]. It is used in skincare for cosmetic reasons and its hydrating and non-irritating properties help to resist ageing in people with sensitive or irritated skin [22]. Moreover, allantoin moisturizes and protects the skin from toxic UV rays. Allantoin helps to rejuvenate cells and removes acne scars [23].

\subsubsection{Nicotinamide}

According to studies, nicotinamide (vitamin B3) is a powerful antioxidant capable of shielding cellular membranes in the brain, which is particularly vulnerable to prooxidants, from oxidative damage caused by reactive oxygen species (ROS) [24]. Nicotinamide (niacinamide) is a water-soluble vitamin B3 (niacin) derivative that is used to prevent and cure niacin deficiency (pellagra). Psychosis, memory loss, and confusion are some of the symptoms, but they can be alleviated by taking niacin supplements. This condition is more prevalent in young people in developing countries such as Africa and India, but it can also affect adults, especially alcoholics who are vitamin deficient or people who have had anorexia nervosa [25]. Nicotinamide is found in tuna, liver, chicken breast, salmon, peanuts, avocado, brown rice, whole wheat, mushrooms, potatoes [26]. Nicotinamide may be a good option for reducing the symptoms of ageing. It is used to treat acne, rosacea, and general skin disorders characterized by redness. Exposure to ultraviolet (UV) radiation, can damage cell DNA and cause melanoma (skin cancer) [27]. It is also recommended for those with osteoarthritis to help with pain, swelling, and joint flexibility. Nicotinamide helps persons with laryngeal cancer reduce tumor development while undergoing radiation, increasing their chances of survival [28].

\section{Material and methods}

\subsection{Chemicals/Materials}

Chemicals used in the study were hydrogen peroxide $\left(\mathrm{H}_{2} \mathrm{O}_{2}\right)$ purchased from Semikem d.o.o, (Sarajevo, Bosnia and Herzegovina, 30\%), phosphate buffer, ascorbic acid (vitamin C) acquired from UFAR d.o.o. (Belgrade, Serbia), potassium dihydrogen phosphate $\left(\mathrm{KH}_{2} \mathrm{PO} 4\right)$ and sodium hydroxide $(\mathrm{NaOH})$ were 
purchased from Centrochem d.o.o (Stara Pazove, Serbia) and Fisher Scientific respectively; distilled water as well as Millipore water. Three samples of phytochemicals (allantoin, betaine and nicotinamide) were obtained from pharmaceutical company „Bosnalijek d.d“ in Sarajevo, Bosnia and Herzegovina.

\subsubsection{Preparation of phosphate buffer (pH7.4)}

$6.8 \mathrm{~g}$ of $\mathrm{KH}_{2} \mathrm{PO}_{4}$ (potassium dihydrogen phosphate) $+1.5 \mathrm{gms}$ of $\mathrm{NaOH}$ (sodium hydroxide) were dissolved in $1000 \mathrm{~mL}$ of millipore water.

\subsubsection{Preparation of 40mM Hydrogen Peroxide}

$4.420 \mathrm{~mL}$ of $\mathrm{H}_{2} \mathrm{O}_{2}$ (30\%) was dissolved in $50 \mathrm{~mL}$ of phosphate buffer (pH7.4) to final $40 \mathrm{mM}$ concentration. Fresh $\mathrm{H}_{2} \mathrm{O}_{2}$ was prepared before the experiment, and put in a dark glass amber volumetric flask. Fresh solution was prepared each time before the experiment.

\subsubsection{Preparation of standard solution (Ascorbic Acid)}

$10 \mathrm{mg}$ of ascorbic acid was dissolved in $10 \mathrm{~mL}$ of phosphate buffer $(\mathrm{pH} 7.4)$ and this solution was used as a standard.

\subsection{Hydrogen peroxide scavenging activity}

The hydrogen peroxide scavenging assay was determined according to Ruch et al method [29]. $10 \mathrm{mg}$ of allantoin, betaine and nicotinamide were weighed on a balance and diluted in $1000 \mu \mathrm{L}$ of distilled water. Allantoin, betaine, and nicotinamide were prepared at different concentrations $(125,250,500$, and 1000 $\mathrm{g} / \mathrm{mL}$ ). In new eppendorf tubes we added $100 \mu \mathrm{L}$ of each sample, and added $400 \mu \mathrm{L}$ of phosphate buffer. Same was performed with the standard solution containing ascorbic acid. Then we added $600 \mu \mathrm{L}$ of $40 \mathrm{mM}$ hydrogen peroxide solution to the tubes, vortexed them and incubated for 10 minutes. We added $100 \mu \mathrm{L}$ of each sample in triplicates in a 96-well plate and measured absorbance at $230 \mathrm{~nm}$ on the Microplate reader (ThermoFisher Scientific, USA) against a blank containing only phosphate buffer without hydrogen peroxide. Ascorbic acid was used as standard/positive control. Samples without hydrogen peroxide were used as a negative control. The abilities to scavenge the hydrogen peroxide were calculated using the (2) equation:

$$
\% \text { scavenged }\left(\mathrm{H}_{2} \mathrm{O}_{2}\right)=\left(A_{o}-A_{1}\right) / A_{o} \times 100
$$

where $A_{o}$ is the absorbance of the control and $A_{l}$ the absorbance of the sample.

The assay was done in triplicates for three times, and results were expressed as mean \pm standard deviation.

\section{Results}

The antioxidant capacity of samples was determined by the decrease in absorbance readings. Decreased absorbance of the reaction mixture indicated increased hydrogen peroxide scavenging activity, meaning an increased level of antioxidant activity. Absorbance's of betaine, allantoin and nicotinamide can be observed in Table 1, 2 and 3 respectively. Furthermore, increased concentration of phytochemicals equals increased $\%$ of $\mathrm{H}_{2} \mathrm{O}_{2}$ inhibition. Higher concentration means higher inhibition of hydrogen peroxide, meaning that the presence of $\mathrm{H}_{2} \mathrm{O}_{2}$ is lowered and that leads us to the conclusion that the chemical showing such results is a good antioxidant. When readings of absorbance's decrease, the concentration is increased and the $\%$ of $\mathrm{H}_{2} \mathrm{O}_{2}$ scavenging activity increases as well. 
Table 1 . Absorbance of betaine vs. concentration range $(125-1000 \mu \mathrm{g} / \mathrm{mL}$

\begin{tabular}{|c|c|}
\hline Concentration $(\boldsymbol{\mu g} / \mathbf{m L})$ & Absorbance of Betaine \\
\hline 125 & 3.743 \\
\hline 250 & 3.740 \\
\hline 500 & 3.736 \\
\hline 1000 & 3.730 \\
\hline
\end{tabular}

Table 2. Absorbance of allantoin with standard deviation (SD) vs. concentration range $(125-1000 \mu \mathrm{g} / \mathrm{mL})$

\begin{tabular}{|c|c|}
\hline Concentration $(\boldsymbol{\mu g} / \mathbf{m L})$ & Absorbance of Allantoin \\
\hline 125 & 3.746 \\
\hline 250 & 3.743 \\
\hline 500 & 3.741 \\
\hline 1000 & 3.734 \\
\hline
\end{tabular}

Table 3. Absorbance of nicotinamide vs. concentration range $(125-1000 \mu \mathrm{g} / \mathrm{mL})$

\begin{tabular}{|c|c|}
\hline Concentration $(\boldsymbol{\mu g} / \mathbf{m L})$ & Absorbance of Nicotinamide \\
\hline 125 & 3.759 \\
\hline 250 & 3.754 \\
\hline 500 & 3.753 \\
\hline 1000 & 3.747 \\
\hline
\end{tabular}

Comparison between absorbances of betaine, allantoin and nicotinamide are represented in a graph form in Figure 1 .
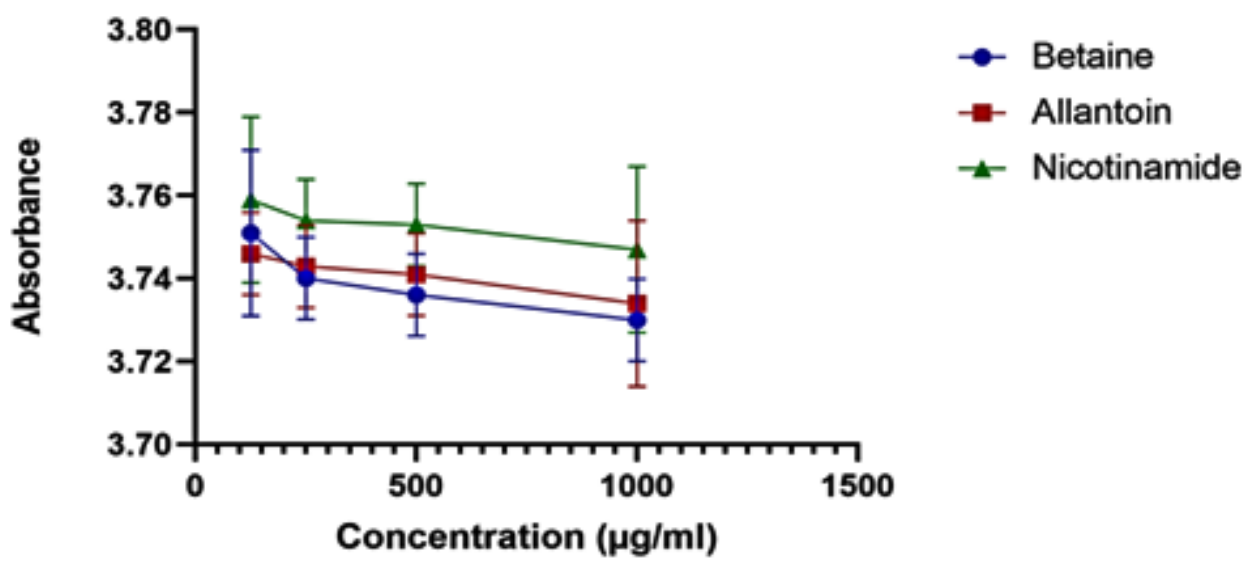

Figure 1. Comparison between the absorbance of samples. The results are presented as mean \pm SD of three experiments.

From the graph in Figure 1, we can see that betaine has the lowest absorbance among other samples, which makes him the one that must have the highest inhibition of hydrogen peroxide. The scavenging ability of betaine, allantoin and nicotinamide on hydrogen peroxide are shown in Table 4 . The scavenging effect of different samples (allantoin, betaine and nicotinamide) on hydrogen peroxide was concentration-dependent $(125-1000 \mu \mathrm{g} / \mathrm{mL})$. 
Table 4. Hydrogen Peroxide Scavenging Activity of the samples vs. concentration

\begin{tabular}{|c|c|c|c|}
\hline $\begin{array}{c}\text { Concentration } \\
(\boldsymbol{\mu g} / \mathbf{m L})\end{array}$ & $\begin{array}{c}\text { Scavenging } \\
\text { activity of the } \\
\text { Betaine (\%) }\end{array}$ & $\begin{array}{c}\text { Scavenging } \\
\text { activity of the } \\
\text { Allantoin (\%) }\end{array}$ & $\begin{array}{c}\text { Scavenging } \\
\text { activity of the } \\
\text { Nicotinamide (\%) }\end{array}$ \\
\hline 125 & 26 & 39 & 6 \\
\hline 250 & 55 & 48 & 18 \\
\hline 500 & 66 & 53 & 23 \\
\hline 1000 & 84 & 71 & 39 \\
\hline
\end{tabular}

According to our results, the betaine was shown to scavenge the $\mathrm{H}_{2} \mathrm{O}_{2}$ radicals with an excellent inhibition percentage of $84 \%$, at the concentration of 1000 micrograms per $\mathrm{mL}$ compared to a standard, being the phytochemical that demonstrated the highest $\mathrm{H}_{2} \mathrm{O}_{2}$ scavenging activity which can be seen on a Figure 1 . At the lowest concentration of 125 micrograms $/ \mathrm{mL}$, betaine had the lowest $\%$ of $\mathrm{H}_{2} \mathrm{O}_{2}$ scavenging activity, accepting the hypothesis that at the highest concentrations there is the highest $\%$ of $\mathrm{H}_{2} \mathrm{O}_{2}$ inhibition. The positive control in this experiment was ascorbic acid.

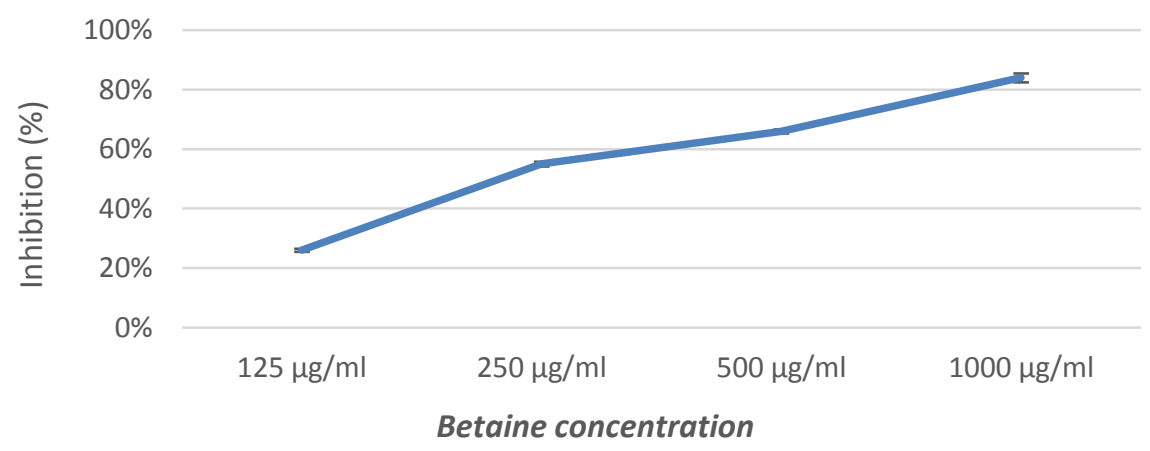

Figure 2. Graph showing $\mathrm{H}_{2} \mathrm{O}_{2}$ scavenging activity of Betaine. The results are represented as mean \pm SD.

Figure 3 illustrates the examined allantoin's capacity to scavenge hydrogen peroxide. The allantoin showed moderate scavenging activity, with exhibition of $71 \%$. Furthermore, the scavenging activity of the nicotinamide is shown in Figure 4. Nicotinamide was discovered to have a very weak inhibition activity with $39 \%$ of the $\%$ of $\mathrm{H}_{2} \mathrm{O}_{2}$ scavenging activity of nicotinamide at the highest concentration of 1000 micrograms $/ \mathrm{mL}$, compared to the allantoin that has $71 \%$ and betaine that has $84 \%$ hydrogen peroxide scavenging activity at the same concentration.

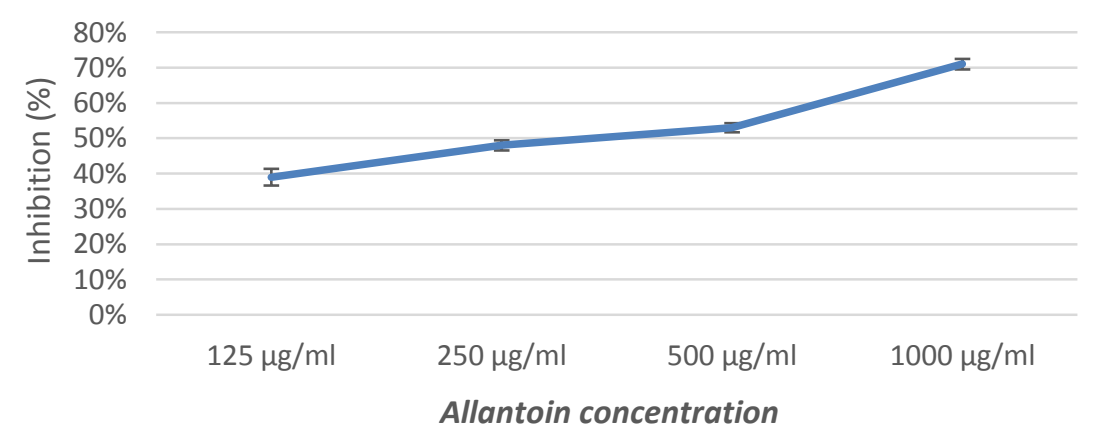

Figure 3. Graph showing $\mathrm{H}_{2} \mathrm{O}_{2}$ scavenging activity of Allantoin. The results are represented as mean \pm SD 


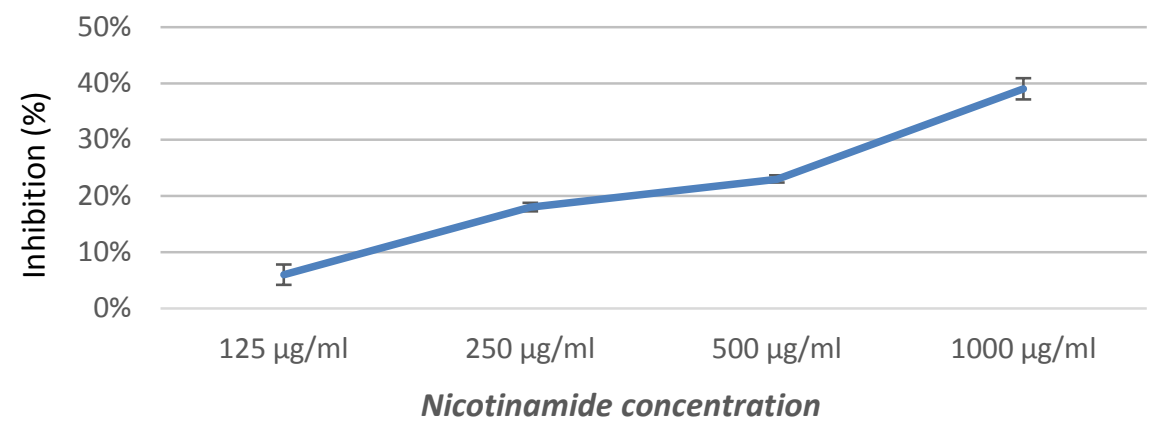

Figure 4. Graph showing $\mathrm{H}_{2} \mathrm{O}_{2}$ scavenging activity of Nicotinamide. The results are represented as mean \pm SD.

The findings revealed that all of the samples possessed $\mathrm{H}_{2} \mathrm{O}_{2}$ scavenging action, which might be attributed to the antioxidant substances, and the presence of phenolic groups that could donate electrons to hydrogen peroxide, thereby neutralizing it into $\mathrm{H}_{2} \mathrm{O}$. Results show that the scavenging activity values on hydrogen peroxide of samples decrease than that of ascorbic acid (vitamin $\mathrm{C}$ ) in the order of ascorbic acid > betaine > allantoin > nicotinamide.

\section{Discussion}

ROS, a reactive oxygen species, are in the spotlight because of their function in a variety of diseases, such as diabetes, arthritis, cancer, ageing, and atherosclerosis. ROS such as oxygen $\left(\mathrm{O}_{2}\right)$, hydrochloric acid $(\mathrm{HOCl})$ and hydrogen peroxide $\left(\mathrm{H}_{2} \mathrm{O}_{2}\right)$ which is renowned for its ability to rapidly penetrate cell membranes can be converted to hydroxyl radicals, a strongly reactive oxidation species, in the presence of transition metal ions such as $\mathrm{Fe}^{2+}$ and possibly $\mathrm{Cu}^{2+}$ ions. Although hydrogen peroxide is not particularly reactive, it has the ability to disable enzymes by oxidizing thiol (- SH) groups. It can be hazardous to cells due to an increase in hydroxyl radicals in the cells [11]. As a result, eliminating $\mathrm{H}_{2} \mathrm{O}_{2}$ from cell or dietary systems is vital for antioxidant defense. Human diseases are caused by an imbalance between oxidative stress and antioxidant defense. So, antioxidant defense supplements may reduce oxidative tissue harm and thereby delay disease development. In our study, the antioxidant effects of allantoin, betaine, and nicotinamide were measured through various biological parameters and compared with a known antioxidant (ascorbic acid). One of the important approaches for measuring the capacity of antioxidants to reduce the number of pro-oxidants such as $\mathrm{H}_{2} \mathrm{O}_{2}$ is to test their $\mathrm{H}_{2} \mathrm{O}_{2}$-scavenging activity. In our study, the $\mathrm{H}_{2} \mathrm{O}_{2}$ - scavenging activity of samples increased in a dose-dependent manner. With increasing concentration, sample absorbance decreased and the efficacy of inhibition raised. Betaine of all three phytochemicals tested (betaine, allantoin and nicotinamide) showed the highest inhibitory action of hydrogen peroxide followed by allantoin and then nicotinamide. The ability of samples to scavenge $\mathrm{H}_{2} \mathrm{O}_{2}$ is due to their phenolics, which may give electrons to $\mathrm{H}_{2} \mathrm{O}_{2}$ and thereby neutralize it to water. Therefore, this study showed that all of the samples possessed $\mathrm{H}_{2} \mathrm{O}_{2}$ scavenging ability, which might be attributed to a significant antioxidant effect of the tested samples.

\section{Conclusions}

In conclusion, allantoin, betaine and nicotinamide based on the results obtained, showed hydrogen peroxide scavenging and powerful antioxidant activities when compared to a standard such as ascorbic acid. The betaine demonstrated the highest scavenging ability, meaning it is the best antioxidant of all the phytochemicals tested in the study. We can find it in foods like beets, spinach, wheat, shellfish, quinoa, brown rice, sweet potatoes and so on. Allantoin was the second-best in scavenging the $\mathrm{H}_{2} \mathrm{O}_{2}$, and it plants that contain it are sugar beet, comfrey, chamomile, tobacco seed, and wheat sprouts. Finally, the third in the ability to scavenge $\mathrm{H}_{2} \mathrm{O}_{2}$ is nicotinamide found in fish, turkey, peanuts, avocado, beef, 
brown rice, etc. The findings of this study suggest that these samples might be utilized as a convenient source of natural antioxidants, as a dietary supplement, or in the pharmaceutical industry. The samples have a high $\mathrm{H}_{2} \mathrm{O}_{2}$ scavenging capability, allowing them to be used in a variety of oxidative stress-related diseases. By scavenging free radicals and thereby avoiding mutagenic modifications and related diseases, the future of dietary antioxidants holds immense promise for ensuring a healthier disease-free lifestyle for mankind.

\section{Abbreviations and acronyms}

ROS reactive oxygen species

$\mathbf{H}_{2} \mathrm{O}_{2}$ hydrogen peroxide

SOD superoxide dismutase

CAT catalase

GPx glutathione peroxidase

$\mathrm{O}_{2} \quad$ oxygen

UV ultraviolet radiation

HOCl hydrochloric acid

$\mathrm{Fe}^{2+} \quad$ iron ion

$\mathrm{Cu}^{2+}$ copper ion

DNA deoxyribonucleic acid

\section{References}

[1] J. F. Turrens, "Mitochondrial formation of reactive oxygen species," The Journal of Physiology, vol. 552, no. 2, pp. 335-344, 2003, doi: https://doi.org/10.1111/j.1469-7793.2003.00335.x.

[2] P. Patlevič, J. Vašková, P. Švorc, L. Vaško, and P. Švorc, "Reactive oxygen species and antioxidant defense in human gastrointestinal diseases," Integr Med Res, vol. 5, no. 4, pp. 250-258, Dec. 2016, doi: 10.1016/j.imr.2016.07.004.

[3] A. E. Fletcher, "Free radicals, antioxidants and eye diseases: evidence from epidemiological studies on cataract and age-related macular degeneration," Ophthalmic Res, vol. 44, no. 3, pp. 191-198, 2010, doi: $10.1159 / 000316476$.

[4] D. B. Zorov, M. Juhaszova, and S. J. Sollott, "Mitochondrial Reactive Oxygen Species (ROS) and ROS-Induced ROS Release," Physiol Rev, vol. 94, no. 3, pp. 909-950, Jul. 2014, doi:

10.1152/physrev.00026.2013.

[5] M. P. Murphy, "How mitochondria produce reactive oxygen species," Biochemical Journal, vol. 417, no. 1, pp. 1-13, Dec. 2008, doi: 10.1042/BJ20081386.

[6] T. Mahaseth and A. Kuzminov, "Potentiation of Hydrogen Peroxide Toxicity," Mutat Res, vol. 773, pp. 274-281, Jul. 2017, doi: 10.1016/j.mrrev.2016.08.006.

[7] G. Pizzino et al., "Oxidative Stress: Harms and Benefits for Human Health," Oxidative Medicine and Cellular Longevity, vol. 2017, pp. 1-13, Jul. 2017, doi: 10.1155/2017/8416763.

[8] M. Hasanuzzaman et al., "Reactive Oxygen Species and Antioxidant Defense in Plants under Abiotic Stress: Revisiting the Crucial Role of a Universal Defense Regulator," Antioxidants, vol. 9, no. 8, p. 681, Jul. 2020, doi: 10.3390/antiox9080681.

[9] B. Tewabe Gebeyehu, "Determination of Caffeine Content and Antioxidant Activity of Coffee," $A J A C$, vol. 3, no. 2, p. 69, 2015, doi: 10.11648/j.ajac.20150302.16.

[10] D. Babu, P. Gurumurthy, S. K. Borra, and K. M. Cherian, "Antioxidant and free radical scavenging activity of triphala determined by using different in vitro models," p. 8 . 
[11] B. Halliwell, M. V. Clement, and L. H. Long, "Hydrogen peroxide in the human body," FEBS Lett, vol. 486, no. 1, pp. 10-13, Dec. 2000, doi: 10.1016/s0014-5793(00)02197-9.

[12] K. Hiramoto, T. Kida, and K. Kikugawa, "Increased Urinary Hydrogen Peroxide Levels Caused by Coffee Drinking.," Biological \& Pharmaceutical Bulletin, vol. 25, no. 11, pp. 1467-1471, 2002, doi: 10.1248/bpb.25.1467.

[13] T. A. V. Beek et al., Chemicals From Plants: Perspectives On Plant Secondary Products. World Scientific, 1999.

[14] C. Forni et al., "Beneficial Role of Phytochemicals on Oxidative Stress and Age-Related Diseases," BioMed Research International, vol. 2019, pp. 1-16, Apr. 2019, doi: 10.1155/2019/8748253.

[15] M. Zhang et al., "Antioxidant Mechanism of Betaine without Free Radical Scavenging Ability," $J$ Agric Food Chem, vol. 64, no. 42, pp. 7921-7930, Oct. 2016, doi: 10.1021/acs.jafc.6b03592.

[16] L. Rivoira, S. Studzińska, M. Szultka-Młyńska, M. C. Bruzzoniti, and B. Buszewski, "New approaches for extraction and determination of betaine from Beta vulgaris samples by hydrophilic interaction liquid chromatography-tandem mass spectrometry," Anal Bioanal Chem, vol. 409, no. 21, pp. 5133-5141, 2017, doi: 10.1007/s00216-017-0461-0.

[17] C. R. Day and S. A. Kempson, "Betaine chemistry, roles, and potential use in liver disease," Biochimica et Biophysica Acta (BBA) - General Subjects, vol. 1860, no. 6, pp. 1098-1106, Jun. 2016, doi: 10.1016/j.bbagen.2016.02.001.

[18] P. M. Ueland, "Choline and betaine in health and disease," J Inherit Metab Dis, vol. 34, no. 1, pp. 315, Feb. 2011, doi: 10.1007/s10545-010-9088-4.

[19] J. R. Hoffman, N. A. Ratamess, J. Kang, S. L. Rashti, and A. D. Faigenbaum, "Effect of betaine supplementation on power performance and fatigue," J Int Soc Sports Nutr, vol. 6, no. 1, p. 7, Dec. 2009, doi: 10.1186/1550-2783-6-7.

[20] “ALLANTOIN.pdf.” Accessed: May 23, 2021. [Online]. Available: https://www.akema.it/pdf/ALLANTOIN.pdf

[21] "What Is Allantoin? Guide to the K-Beauty Ingredient for Sensitive Skin | Allure." https://www.allure.com/story/what-is-allantoin-skin-care-ingredient-korean-beauty (accessed May 23, 2021).

[22] "Spotlight on Allantoin: What Is It and Can It Benefit Your Skin?," skinbetter science ${ }^{\circledR}$, Feb. 20, 2017. https://skinbetter.com/spotlight-allantoin-can-benefit-skin/ (accessed May 23, 2021).

[23] “Allantoin: A Safe \& Effective Skin Care Ingredient You Need To Know About," SkinKraft. https://skinkraft.com/blogs/articles/allantoin-skin-benefits (accessed May 23, 2021).

[24] J. P. Kamat and T. P. Devasagayam, "Nicotinamide (vitamin B3) as an effective antioxidant against oxidative damage in rat brain mitochondria," Redox Rep, vol. 4, no. 4, pp. 179-184, 1999, doi: $10.1179 / 135100099101534882$.

[25] M. Knip et al., "Safety of high-dose nicotinamide: a review," Diabetologia, vol. 43, no. 11, pp. 13371345, Oct. 2000, doi: 10.1007/s001250051536.

[26] "16 Foods That Are High in Niacin (Vitamin B3)," Healthline, Oct. 05, 2018. https://www.healthline.com/nutrition/foods-high-in-niacin (accessed May 24, 2021). 
BES Vol. 2, No. 1, June 2021, pp.1-10

[27] A. C. Chen and D. L. Damian, "Nicotinamide and the skin: Nicotinamide and the skin," Australasian Journal of Dermatology, vol. 55, no. 3, pp. 169-175, Aug. 2014, doi: 10.1111/ajd.12163.

[28] "Niacinamide: MedlinePlus Supplements.” https://medlineplus.gov/druginfo/natural/1534.html (accessed May 23, 2021).

[29] R. J. Ruch, S. J. Cheng, and J. E. Klaunig, "Prevention of cytotoxicity and inhibition of intercellular communication by antioxidant catechins isolated from Chinese green tea," Carcinogenesis, vol. 10, no. 6, pp. 1003-1008, Jun. 1989, doi: 10.1093/carcin/10.6.1003. 